\title{
Illthrift in Suckling Lambs Secondary to Umbilical Infections and Possible Implication of Cryptosporidiosis as a Risk Factor
}

\author{
Nektarios D. Giadinis, ${ }^{1}$ Elias Papadopoulos, ${ }^{2}$ Panayiotis Loukopoulos, ${ }^{3}$ Nikolaos Panousis, ${ }^{1}$ \\ Emmanouil Kalaitzakis, ${ }^{4}$ Asimakis Koutsoumpas, ${ }^{1}$ and Harilaos Karatzias ${ }^{1}$ \\ ${ }^{1}$ Clinic of Farm Animals, Faculty of Veterinary Medicine, Aristotle University of Thessaloniki, 54627 Thessaloniki, Greece \\ ${ }^{2}$ Laboratory of Parasitology and Parasitic Diseases, Faculty of Veterinary Medicine, Aristotle University of Thessaloniki, \\ 54627 Thessaloniki, Greece \\ ${ }^{3}$ Laboratory of Pathology, Faculty of Veterinary Medicine, Aristotle University of Thessaloniki, 54627 Thessaloniki, Greece \\ ${ }^{4}$ Clinic of Medicine, Faculty of Veterinary Medicine, University of Thessaly, 43100 Karditsa, Greece
}

Correspondence should be addressed to Nektarios D. Giadinis, ngiadini@vet.auth.gr

Received 6 February 2011; Accepted 30 March 2011

Academic Editor: Guillermo Virkel

Copyright () 2011 Nektarios D. Giadinis et al. This is an open access article distributed under the Creative Commons Attribution License, which permits unrestricted use, distribution, and reproduction in any medium, provided the original work is properly cited.

\begin{abstract}
Illthrift was observed in 20/60 lambs aged 40-45 days in a dairy sheep flock in Greece. Cryptosporidiosis had been diagnosed and successfully treated with halofuginone lactate a month earlier. Parasitological examinations were negative for endoparasites while hematology and biochemistry were unremarkable. Necropsy of 5 lambs revealed lung and liver abscessation, presumably secondary to umbilical infections due to poor farm hygiene, though umbilical lesions were not observed. No new cases were observed following treatment of the umbilicus of newborn lambs with chlorexidine. Although umbilical infections are common, this is the first reported case of illthrift in lambs attributed to umbilical infection; illthrift may be the only clinical manifestation of such infections. The prior presence of cryptosporidiosis may have contributed to the severity of the infection through the reduction of local immunity. Recognition of this possibly underdiagnosed or underappreciated condition may improve medical, production, and welfare standards in the sheep industry.
\end{abstract}

\section{Introduction}

A range of factors can cause illthrift in lambs, through various mechanisms; these factors include malnutrition, trace element and vitamin deficiencies, parasitic infections, orf, lameness, and chronic suppurative infections, especially of the respiratory system [1-4].

Chronic suppurative infections in lambs usually originate from the digestive system, or from respiratory, cutaneous, or umbilical infections [1]. Umbilical infections are encountered frequently in practice and usually result in liver abscessation but may also lead to septic peritonitis, localized fibrinous peritonitis, or polyarthritis. These conditions are usually accompanied by anorexia and mortality [5-7].

Although umbilical infections can cause illthrift in calves [1], they have not been reported to date to cause illthrift in lambs, despite being commonly encountered in this species $[3,7]$. This short communication describes a case of illthrift in lambs of a dairy sheep flock. The illthrift was attributed to umbilical infection and responded to the improvement of the farm's hygiene.

\section{Case Report}

The owner of a dairy sheep flock in northern Greece, consisting of 300 Chios $\times$ East Friesian sheep reared under the semi-intensive feeding system, complained initially for high incidence of diarrhea and mortality (approx. 30\% and $20 \%$, resp.) in lambs aged 7-10 days. Three diarrheic lambs were admitted to the Clinic of Farm Animals for examination. Following parasitological, microbiological, and clinicopathological examinations, a diagnosis of cryptosporidiosis was established. Subsequently, all the lambs in the flock (both diarrheic and nondiarrheic) that were less than 15 days old were treated with the anticryptosporidial drug halofuginone lactate $(100 \mu \mathrm{g} / \mathrm{kg}$ b.w./day per os) for 7 
days (Halocur-Intervet) and both diarrhea incidence and mortality ceased.

One month later the farmer complained that 20 out of 60 lambs had growth retardation. In fact, although they were 40-45 days old, on examination they weighed about $8 \mathrm{~kg}$ instead of $13 \mathrm{~kg}$ which is the expected weight based on their age and breed standards. Treatment of all affected lambs with a combination of penicillin and streptomycin was ineffective. Three 45 -day-old lambs that showed growth retardation were admitted to the Clinic of Farm Animals for examination. They were alert, afebrile, and slightly inappetent. Fecal parasitological examinations were negative for coccidians and other endoparasites. Hematology revealed slight leukocytosis, while their biochemical profile was normal. All three lambs were euthanized. Necropsy revealed the presence of multiple abscesses in the liver and lungs, and focal adhesions of the lungs to the pleura. Similar necropsy findings were observed in two more lambs that were examined on a subsequent visit to the farm; extensive consolidation mainly of the caudal portion of the lungs was also observed in one of these lambs (Figures 1 and 2). Concerning the assessment of the farm's hygiene conditions, bad sanitation was observed in the newborns' pens during our visit. Moreover, the farmer informed us that he had never cleaned or disinfected the umbilicus of newborn lambs.

Based on the history, clinical, and necropsy findings, illthrift was presumably attributed to the presence of lung and liver abscesses secondary to umbilical infection. Following our recommendations, the farmer started to disinfect the umbilicus of the remaining newborn lambs with a chlorhexidine solution and the cases of illthrift ceased as a result, as 150 lambs that were born after the application of disinfection were healthy and had a normal growth rate. The initial presumed cause of illthrift was therefore consistent with and indirectly confirmed by the clinical outcome.

\section{Discussion}

Although umbilical infection is considered as a possible cause of illthrift in calves [1], this is the first reported case of illthrift in lambs secondary to umbilical infection. This infection can lead to internal abscessation of the liver through the portal vein, as well as of other internal organs. In these cases, illthrift is the result of bacterial toxaemia $[1,4]$. It is generally accepted that the most commonly implicated microorganisms in lamb navel-ill syndrome are Escherichia coli, Arcanobacterium pyogenes, and Streptococcus dysgalactiae [5]. Unfortunately, bacterial cultures were not conducted in the present study, since the animals had received antibiotics prior to their admission to the clinic.

Other conditions that may cause illthrift in lambs are trace element and vitamin deficiencies $[1,8]$, nephrosis, lameness, coccidiosis, and other parasitic infections $[3,4$, $7,9]$. The clinicopathological findings, as well as the parasitological examinations, excluded the presence of all these conditions. Cryptosporidiosis, which was initially diagnosed in this flock, has been reported as a possible cause of illthrift by some authors [10, 11]. Although our research group has not found any correlation of cryptosporidiosis, as an

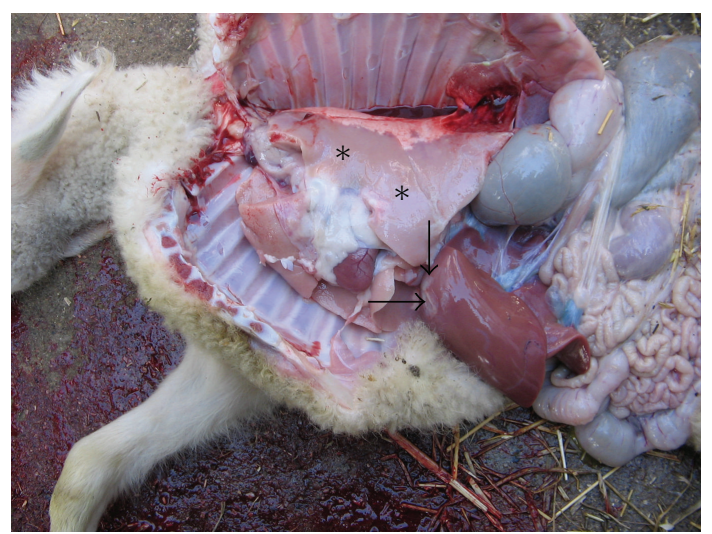

Figure 1: Necropsy revealed the presence of abscesses in the liver (arrows) and lungs of all five lambs necropsied. In the lamb shown here, extensive consolidation, mainly of the caudal portion of the lungs, was also observed (asterisks).

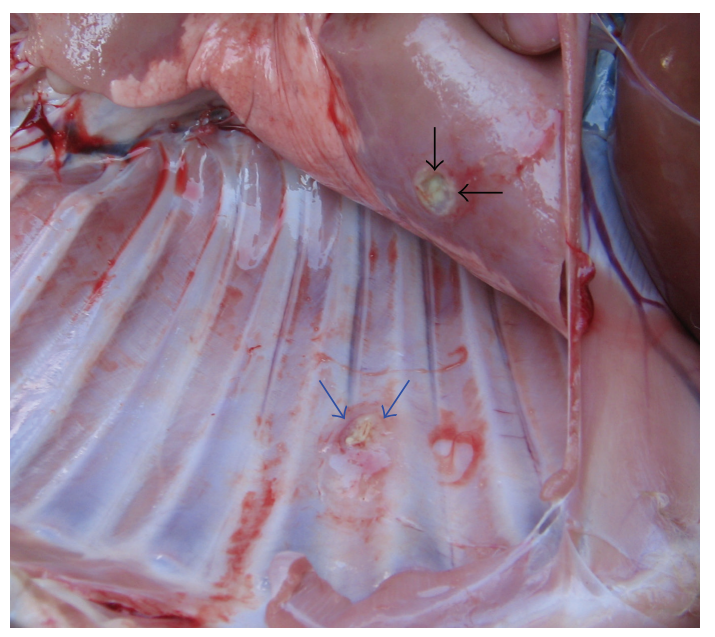

Figure 2: Close up of the chest cavity of the lamb in Figure 1. An abscess (black arrows) in the lungs and focal adhesions of the lungs to the pleura (blue arrows, ruptured postmortem) are shown.

individual entity, with growth retardation in lambs [12] and, furthermore, the cryptosporidial infection initially present was eliminated following treatment with halofuginone lactate $[10,12,13]$, the initial presence of cryptosporidiosis may have contributed to the severity of the umbilical infections and their sequelae.

It has been shown that IgA, responsible for local immunity, plays an important role in the protection of lambs against cryptosporidiosis; there is high aggregation of specific IgA in intestinal secretions, while specific IgM and IgG antibodies are first detected in serum seven days after infection [14]. The above suggests that the concentration of host antibodies on the intestinal wall and their absence or decrease in the umbilicus area allowed the massive increase of pathogens, present due to bad hygienic conditions, and the development of heavy infection and severe sequelae. A similar situation exists during the periparturient rise in egg worm output in ewes. It has been shown that relaxation of 
immunity, which allows the worm and egg rise, is expressed by a reduction in circulating eosinophils and plasma total antibody (Tab) and lower titres of Tab and IgG in the small intestine of pregnant ewes. Circulating eosinophils and plasma Tab remain low in suckled ewes, and at the local level, lactating ewes also have lower titres of Tab, IgG, IgM, $\mathrm{IgA}$, and $\mathrm{IgE}$ as well as fewer mast cells, globule leucocytes and goblet cells in intestinal tissue [15]. Another factor that could predispose to umbilical infections is the reduced passive immunity and overall immune status due to reduced nutritional uptake secondary to cryptosporidiosis-associated malabsorptive enteropathy $[1,6]$.

The diagnosis was based on history and necropsy findings. However, the attribution of illthrift to umbilical infection was initially presumptive, as lesions in the umbilicus were not observed, a finding that is not uncommon in umbilical infections, since necropsies are performed later in the clinical course of disease $[1,6]$. This was subsequently indirectly confirmed by the response to umbilical disinfection immediately after birth, namely, the lack of new cases of illthrift.

\section{Conclusion}

From the present findings, umbilical infections may result in reduced growth rate in lambs due to the causation of lung and liver abscessation, and illthrift may be the only clinical manifestation of such infections. Recognition of this condition, which may be underdiagnosed or underappreciated, may help improve medical, production, and welfare standards in the industry.

\section{References}

[1] O. M. Radostits, C. C. Gay, K. W. Hinchcliff, and P. D. Constable, Veterinary Medicine, Saunders Elsevier, Oxford, UK, 10th edition, 2007.

[2] M. Sheehan, J. P. Cassidy, J. Brady et al., "An aetiopathological study of chronic bronchopneumonia in lambs in Ireland," Veterinary Journal, vol. 173, no. 3, pp. 630-637, 2007.

[3] N. Sargison, Sheep Flock Health, Blackwell, Oxford, UK, 2008.

[4] N. D. Giadinis, P. Loukopoulos, P. Tsakos, M. KritsepiKonstantinou, E. Kaldrymidou, and H. Karatzias, "Illthrift in suckling lambs attributed to lung pyogranuloma formation," Veterinary Record, vol. 165, no. 12, pp. 348-350, 2009.

[5] G. H. Watkins and M. W. Sharp, "Bacteria isolated from arthritic and omphalatic lesions in lambs in England and Wales," Veterinary Journal, vol. 156, no. 3, pp. 235-238, 1998.

[6] C. B. Navarre and D. G. Pugh, "Diseases of the gastrointestinal system," in Sheep and Goat Medicin, D. G. Pugh, Ed., pp. 69105, Saunders, Philadelphia, Pa, USA, 2002.

[7] P. R. Scott, Sheep Medicine, Manson, London, UK, 2007.

[8] E. J. Underwood and N. F. Suttle, The Mineral Nutrition of Livestock, CABI Publishing, Oxfordshire, UK, 3rd edition, 2004.

[9] J. C. Hidson and A. C. Winter, Outline of Clinical Diagnosis in Sheep, Wright, London, UK, 1990.

[10] M. Naciri and P. Yvore, "Efficiency of halofuginone lactate in the treatment of experimental cryptosporidiosis in lambs," Recuell de Medecine Veterinaire, vol. 165, pp. 823-826, 1989.
[11] N. Naciri, S. Bareille, R. Fournier, and W. G. Holland, "Use of halofuginone in the control of Cryptosporidium parvum infections in sheep," in Proceedings of the 6th International Sheep Veterinary Congress, pp. 257-258, Crete, Greece, 2005.

[12] N. D. Giadinis, E. Papadopoulos, N. Panousis, M. Papazahariadou, S. Q. Lafi, and H. Karatzias, "Effect of halofuginone lactate on treatment and prevention of lamb cryptosporidiosis: an extensive field trial," Journal of Veterinary Pharmacology and Therapeutics, vol. 30, no. 6, pp. 578-582, 2007.

[13] A. C. Causape, C. Sanchez-Acedo, J. Quilez, E. Del Cacho, and M. Viu, "Efficacy of halofuginone lactate against natural Cryptosporidium parvum infections in lambs," Research and Reviews in Parasitology, vol. 59, pp. 41-46, 1999.

[14] A. M. Beasley, L. P. Kahn, and R. G. Windon, "The periparturient relaxation of immunity in Merino ewes infected with Trichostrongylus colubriformis: parasitological and immunological responses," Veterinary Parasitology, vol. 168, no. 1-2, pp. 60-70, 2010.

[15] B. D. Hill, D. A. Blewett, A. M. Dawson, and S. Wright, "Analysis of the kinetics, isotype and specificity of serum and coproantibody in lambs infected with Cryptosporidium parvum," Research in Veterinary Science, vol. 48, no. 1, pp. 76$81,1990$. 

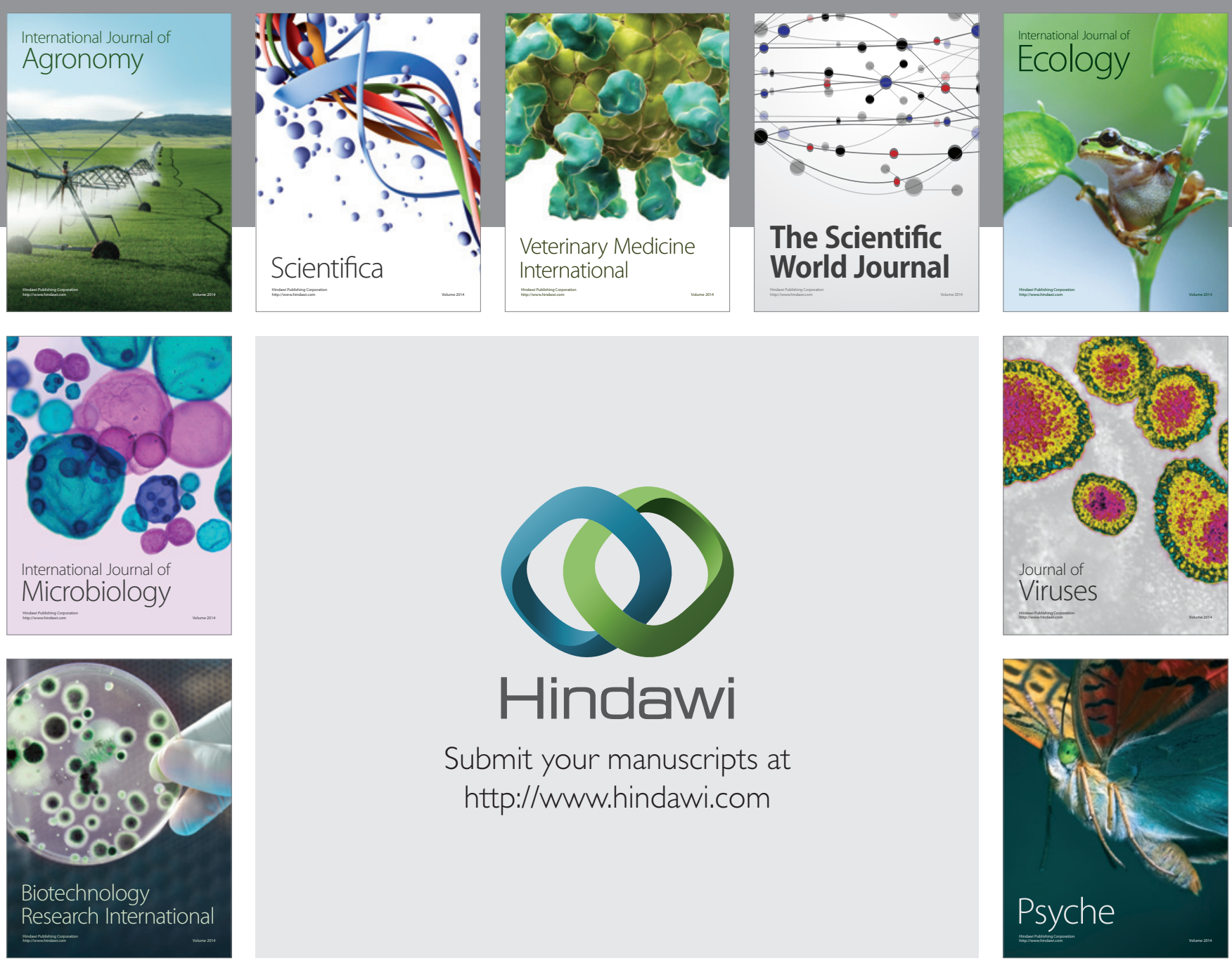

Submit your manuscripts at

http://www.hindawi.com
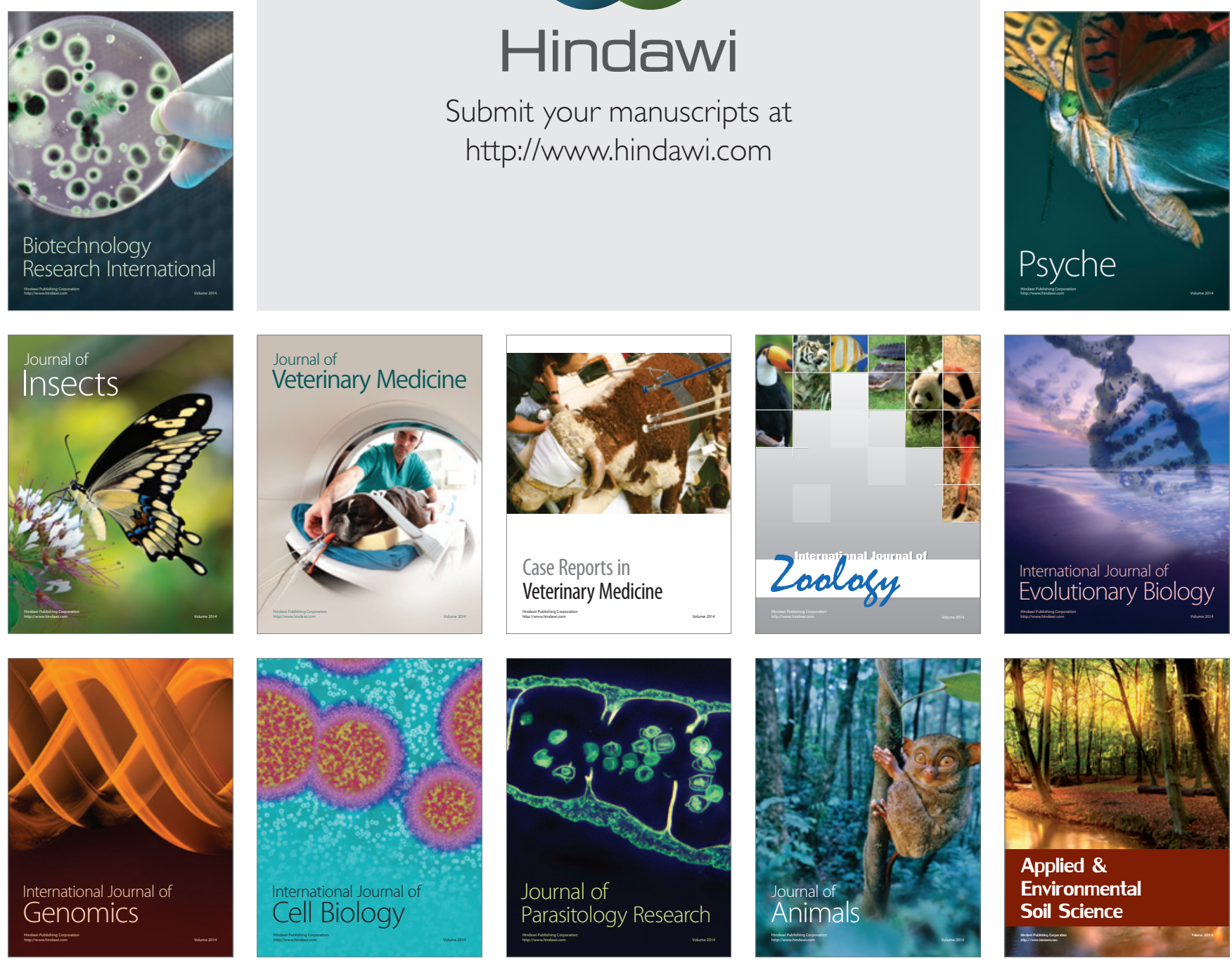